largely for the referenced values of the same feed. The present communication focuses on some of the issues which appear in predicting feed values of tropical resources for ruminants. Other important aspects, such as chemical analysis, have been discussed in the plenary session.

Are in vitro and in vivo data equivalent? A first aspect adresses the level of equivalence between information from in vitro and in vivo data. In vivo digestibility of organic matter (OMD) is the key information to assess the feed nutritive value. However, it is fairly difficult and costly to carry out OMD, therefore in vitro methods are more and more frequently applied. From pooling data of the literature, it appears that in vitro and in vivo results are rather similar for feed having NDF content and OM digestibility in the ranges of $40-50 \% \mathrm{DM}$ and $55-65 \%$ respectively. For higher NDF contents, in vivo OMD values become higher than in vitro ones with differences that can be more than 20 points. This bias is probably the consequence of the adaptation of animals to rough feed (longer transit and chewing times, more $\mathrm{N}$ recycling...). Consequently, in vitro data must be used cautiously to predict in vivo OMD for feeds rich in the cell wall.

Usefulness of in sacco data? A second issue concerns the usefulness of values of in sacco, or in situ, degradation of feed constituents. This method has proved to be interesting to predict protein or starch digestion in the rumen and by-pass flows of the corresponding fractions of feed. Thus, a challenge is to pool the published in situ data to extract main values allowing the building of tables including reference values of in sacco effective degradability of $\mathrm{N}$, starch.... Moreover, since dietary indigestible NDF is the major determinant of OMD, the in sacco method can also be applied to predict NDF undigestibility. Comparison between in sacco and in vivo NDF undigestibility of rations is very encouraging. Thus in sacco can also be used to rank concentrate and by-product feed according to their (un)digestibility of NDF. This approach distinguishes feeds in 2 extreme groups according to their NDF undigestibility: more than $50 \%$ (cereal straws, hulls of rapeseed, peanut and sunflower...) and less than $20 \%$ (palm products, corn grain products, soybean hulls, citrus and beet pulp...). Only a few feed have an intermediary position between these two groups.

From feed to diet evaluation? A third aspect deals with the fact that tabulated nutritive values (NV) of feeds are assessed in standard conditions while, in practice, the target is to evaluate NV of diets. Diet NV is not the sum of the associated feed NV due to influences of various factors: feeding level (FL in terms of DMI\%LW), percentage of concentrate (\%CO), level of $\mathrm{N}$ supply to microbes in the rumen... Moreover for some items such as $\mathrm{CH} 4$, the influence of $\mathrm{FL}$ and \% $\% \mathrm{CO}$ are interacting and complicated, demonstrating that $\mathrm{CH} 4$ production cannot be a tabulated feed attribute. Thus, the prediction of dietary NV from feed is a complicated task requiring response functions to key factors of not only OMD or energy digestibility, but also energy flow like $\mathrm{CH} 4$ and urine. Estimation and standardization of these functions of response is an important issue for the future.

From nutritive to feeding values? Feeding value is generally assessed with the level of spontaneous DOM intake per $\mathrm{kgLW}^{0.75}$, thus it is approximatively the product of DMI $(\mathrm{kg} /$ $\mathrm{LW}^{0.75}$ ) and OMD which are also mutually linked. For forages, since the DMI values referenced in tables are measured into cages, an ultimate concern is the prediction of the actual value of DMI at pasture which could be somewhat different.

In conclusion, the contexts presented demonstrate that nutritive and feed value evaluation from the numerous available data has to be rigourously conducted and must be carefully traced to allow further improvements without having to re-start from zero.

\title{
Measurement methods for generating new data, the example of NIRS
}

\author{
Rob M Dixon ${ }^{1}$ and David B Coates ${ }^{2}$
}

\footnotetext{
${ }^{1}$ The University of Queensland, QAAFI, PO Box 6014, Rockhampton 4702, Australia; ${ }^{2}$ ATSIP, CSIRO Ecosystems Sciences, PMB PO Aitkenvale, Qld 4814, Australia Email : Rob.Dixon@deedi.qld.gov.au
}

Introduction Near infrared (NIR) spectroscopy is an analytical technique measuring light absorption in the $780-2500 \mathrm{~nm}$ region which is closely related to important chemical bonds $(\mathrm{OH}, \mathrm{NH}$ and $\mathrm{CH})$. NIR can be used to measure many nutritionally important constituents of concentrate and forage feedstuffs (Roberts et al. 2004; Andres et al. 2005).

Approach NIR spectroscopy depends on the development, in representative sets of samples, of mathematical relationships (calibration equations) between spectra and constituents or attributes of the samples measured by conventional chemistry. These calibrations are then applied to the spectra of unknown samples to estimate constituents or attributes of interest (Williams and Norris, 2001). NIR calibration equations tend to be specific to the circumstances of the data used for their development.

A major advantage of NIRS is that application allows rapid, routine and economical analysis of feedstuffs where appropriate calibrations are available for constituents of interest. Also, 
only one determination of the NIR spectrum of the feedstuff is required to estimate a large range of constituents. NIR can also be used to measure some functional properties of feedstuffs. Disadvantages include the need to acquire and analyse large (hundreds or sometimes thousands) sets of samples representing the range of NIR spectral diversity associated with various classes of the feedstuff (e.g. plant cultivars, agronomic, soil and seasonal conditions) to develop reliable and robust calibration equations. Conventional analysis is required for each constituent or attribute to determine the reference values necessary to develop the calibration equations. In addition, ongoing conventional analysis of subgroups of samples is needed indefinitely to validate and adjust calibration equations. Other constraints are that NIR instrumentation requires substantial capital investment, and considerable technical skills are required to develop and maintain calibration equations.

In a similar manner to the analysis of forages, NIR analysis of faeces allows estimation of many attributes of the diet of ruminants. Such estimation of diet from faecal NIR spectra depends on the similarity of the NIR spectra of forages and matching faeces (diet-faecal pairs) despite effects of digestion in the gastro-intestinal tract. Prediction of diet from faecal NIR spectra appears most reliable for forage diets. Thus many attributes of the diet selected by grazing ruminants (e.g. nitrogen content, digestibility, non-grass content) can be estimated when appropriate calibration equations are available.

In general NIRS is appropriate for organic constituents that comprise greater than about $1 \%$ of the feedstuff. NIRS is not generally suitable for mineral analysis although there are exceptions. Some functional as well as chemical properties of feedstuffs can be measured using NIRS. For example NIRS is often more satisfactory than the established laboratory procedures to estimate in vitro digestibility of forages. Also the voluntary intake of forage dry matter and digestible energy by ruminants can often be estimated more accurately from NIRS measurements of the forage than from constituents such as fibre or lignin.

Conclusions NIRS can be used to analyse many chemical and functional properties of animal feedstuffs. Reliable analysis depends on development and maintenance of appropriate calibration equations, and these require appreciable resources and skills.

\section{References}

Andres S, Murray I, Calleja A and Giraldez FJ 2005. J Near Spectroscopy 13, 301-311. Roberts CA, Workman J and Reeves JB 2004. Near-Infrared Spectroscopy in Agriculture. Agronomy Monograph No 44. American Society of Agronomy, Madison, Wisconsin, USA.

Williams P and Norris K 2001. Near-Infrared Technology in the Agricultural and Food Industries, 2nd Edn. American Association of Cereal Chemists, St Paul, Minnesota, USA

\title{
Australian Data for a Tropical Feeds Database
}

\author{
Peter R Martin ${ }^{1}$ and Rob M Dixon ${ }^{2}$ \\ ${ }^{1}$ DEEDI, Health and Food Sciences Precinct PO Box 156, Archerfield BC QLD 4108, Australia; ${ }^{2}$ The University of Queensland, QAAFI, PO Box 6014, \\ Rockhampton 4702, Australia \\ Email : Peter.Martin@deedi.qld.gov.au
}

Introduction: A public domain database providing information on tropical feeds would be valuable to livestock farmers and researchers both in Australia and internationally. The Animal Science Nutrition Laboratory of the Department of Employment, Economic Development and Innovation (DEEDI), Queensland Government, operates a central laboratory to provide an extensive range of nutritional analyses of pastures and other animal feedstuffs. Archival records date back more than 30 years. Data since 1995 has been stored in a digital format.

Detail: A Microsoft Access database currently contains about 10,000 records. This is divided about equally between forages (pasture samples immediately oven-dried or forages conserved as hay or silage) and concentrates (mainly grains or by-products of cereals or legumes, and oilseed meals).

For data storage most of the samples have been categorised and named as described by Göhl (1975). The number of records within each of these categories is shown in Table 1. Where information is available entries are further described according to common name, variety, part

Table 1 Categories and number of entries within the Animal Science database

\begin{tabular}{llll}
\hline \hline Code & Category & Description & Number \\
\hline A & Grass & Grasses and mixed pastures & 2959 \\
B & Legume & Pasture and browse legumes & 649 \\
C & Forage & Miscellaneous forage and & 1203 \\
& & browse plants \\
D & Fruit & Fruits and vegetables & 18 \\
E & Root & Root crops & 118 \\
F & Cereal & Cereal grains & 2723 \\
G & Oilseed & Oilseeds, grain, meals and cakes & 1211 \\
H & Animal Origin & Feeds of animal origin & 901 \\
I & Misc Feed & Miscellaneous feedstuffs & 423 \\
K & Premixes & Defined feed additives & 30 \\
\hline \hline
\end{tabular}

